\title{
Erratum
}

\section{(1) Preferred longitudes in sunspot activity (2) Preferred sunspot longitudes: non-axisymmetry and differential rotation}

\author{
I. G. Usoskin ${ }^{1}$, S. V. Berdyugina ${ }^{2,3}$, and J. Poutanen ${ }^{4}$ \\ 1 Sodankylä Geophysical Observatory (Oulu unit), PO Box 3000, 90014 University of Oulu, Finland \\ e-mail: ilya.usoskin@oulu.fi \\ 2 Institut für Astronomie, ETH Zentrum, 8092 Zürich, Switzerland \\ 3 Tuorla Observatory, University of Turku, 21500 Piikkiö, Finland \\ 4 Astronomy Division, PO Box 3000, 90014 University of Oulu, Finland \\ (1) A\&A, 405, 1121-1128 (2003), DOI:10.1051/0004-6361:20030748 \\ (2) A\&A, 441, 347-352 (2005), DOI:10.1051/0004-6361:20053201
}

Key words. Sun: activity - Sun: magnetic fields - errata, addenda

We correct three errors which appeared in these closely related papers.

1. In Paper (1), we were primarily interested in the effect of the differential rotation rate $B$ and neglected the difference between the equatorial rotation rate $A$ and the Carrington rotation rate in Eq. (5), which results in a slope in Figs. 3 and 4 that is too steep. This was corrected without comment in Paper (2) and subsequent papers.

2. In Paper (2), Eq. (3) contains a typographical error, with $\Omega_{j}$ and $\Omega_{\mathrm{C}}$ interchanged. It should read

$$
\Lambda_{i}=\Lambda_{0}+T_{\mathrm{C}} \sum_{j=N_{0}}^{i}\left(\Omega_{j}-\Omega_{\mathrm{C}}\right) .
$$

This correct form was used in all numerical work in that paper and all subsequent papers (e.g., Berdyugina et al. 2006, A\&A, $445,703)$.

3. In Paper (2), the given form of Eq. (6) is incorrect. It should read

$$
\epsilon_{k i}=\min \left(\left|\tilde{\lambda}_{k i}\right| ;\left|\tilde{\lambda}_{k i}-180^{\circ}\right| ;\left|\tilde{\lambda}_{k i}-360^{\circ}\right|\right) \text {. }
$$

This correct form was used in all numerical work in that and subsequent papers. 